\title{
O CUIDADOR DA PESSOA IDOSA: PROCESSO EDUCATIVO OU FORMAÇÃO PARA O MERCADO?
}

\author{
THE CARE OF ELDERLY PEOPLE: EDUCATIONAL PROCESS \\ OR TRAINING FOR THE MARKET?
}

\section{EL CUIDADOR DE PERSONAS MAYORES: ¿PROCESO EDUCATIVO O FORMACIÓN PARA EL MERCADO?}

\author{
Alcione Assunção Correia Lima ${ }^{1}$ \\ Programa de Pós-graduação em Gestão e Tecnologia Aplicadas \\ à Educação- GESTEC- Universidade do Estado da Bahia-UNEB-BA-Brasil. E-mail: \\ alcioneassuncaolima@gmail.com \\ Orcid: https://orcid.org/0000-0002-3815-3083 \\ Fernando Luís de Queiroz Carvalho ${ }^{2}$ \\ Programa de Pós-graduação em Gestão e Tecnologia Aplicadas \\ à Educação- GESTEC- Universidade do Estado da Bahia-UNEB-BA-Brasil. \\ E-mail: fcarvalho@uneb.br \\ Orcid: https://orcid.org/0000-0003-1997-2881 \\ José Raimundo Oliveira Lima ${ }^{3}$ \\ Programa de Pós-Graduação em Planejamento Territorial - \\ PLANTER-Universidade Estadual de Feira de Santana \\ (UEFS)-Feira de Santana-BA-Brasil. \\ E-mail: zeraimundo@uefs.br \\ Orcid: https://orcid.org/0000-0002-3646-307x
}

\begin{abstract}
Resumo: As estratégias de educação em saúde têm sido mecanismos bastante efetivos e promissores no cuidado com a pessoa idosa, cuja base é a articulação de um trabalho educativo entre cuidador, paciente e família. Este estudo objetiva discutir o ofício de cuidador da pessoa idosa no desenvolvimento de suas habilidades frente às novas relações de trabalho, bem como as configurações mercadológicas em que o cuidado com o outro passa assumir um "valor de troca" ou define-se num trabalho como outro qualquer oferecido no mercado. Tratase de um estudo de campo, exploratório, de caráter qualitativo, realizado no município de Feira de Santana-BA, cuja população estima-se em 591.707 habitantes e a população idosa corresponde a 8,7\%, conforme censo do IBGE (2010). Espera-se contribuir para a reflexão sobre as práticas do profissional "cuidador de pessoas idosas", cuja função tem se aproximado
\end{abstract}

\footnotetext{
1 Mestranda em Gestão e Tecnologias Aplicadas a Educação pela Universidade do Estado da Bahia.

2 Prof. Dr. Titular da Universidade do Estado da Bahia.

3 Prof. Dr. Titular da Universidade Estadual de Feira de Santana.
} 
de uma tendência mercadológica, caracterizando-se como um serviço, uma mercadoria como outra qualquer, um valor de troca.

Palavras-chave: Pessoa idosa. Saúde e educação. Mercado de trabalho.

Abstract: Health education strategies have been very effective and promising mechanisms in caring for the elderly, whose basis is the articulation of an educational work between caregiver, patient and family. This study aims to discuss the profession of caregiver of the elderly in the development of their skills in the face of new work relationships, as well as the market configurations in which the care for others starts to assume an "exchange value" or is defined in a job any other offered on the market. This is a qualitative, exploratory field study carried out in the municipality of Feira de Santana-BA, whose population is estimated at 591.707 inhabitants and the elderly population corresponds to $8.7 \%$, according to the IBGE census (2010). It is expected to contribute to the reflection on the practices of the professional "caregiver of elderly people", whose function has been approaching a market trend, characterized as a service, a commodity like any other, an exchange value.

Keywords: Caregiver of the elderly. Health education. Work process.

Resumen: Las estrategias de educación en salud han sido mecanismos muy efectivos y prometedores en el cuidado del anciano, cuya base es la articulación de una labor educativa entre cuidador, paciente y familia. Este estudio tiene como objetivo discutir la profesión de cuidador de personas mayores en el desarrollo de sus habilidades ante las nuevas relaciones laborales, así como las configuraciones de mercado en las que el cuidado de los demás comienza a asumir un "valor de cambio" o se define en un puesto de trabajo como cualquier otro ofrecido en el mercado. Se trata de un estudio cualitativo, exploratorio de campo realizado en el municipio de Feira de Santana-BA, cuya población se estima en 591.707 habitantes y la población anciana corresponde al 8,7\%, según el censo del IBGE (2010). Se espera contribuir a la reflexión sobre las prácticas del profesional "cuidador de personas mayores", cuya función ha ido acercándose a una tendencia de mercado, caracterizada como un servicio, una mercancía como cualquier otra, un valor de cambio.

Palabras Clave: Mayor. Salud y educación. Mercado de trabajo.

Data de recebimento: $27 / 08 / 2020$

Data de aprovação: 20/12/2020 


\section{1 - INTRODUÇÃO}

Ontologicamente, o homem sempre foi responsável pela produção da sua existência material ou pela reprodução da vida. Neste particular, seu dispêndio de energia física ou intelectual configura-se como trabalho que tem assumido, ao longo dessa história, formatos diversos. Entretanto, esses formatos têm se consubstanciado em duas posições fundamentais: uma, enquanto se compreende o trabalho como um processo educativo e político, em que suas reflexões e orientações determinam uma dimensão em sua vida; outra, quando somos formados por outros, para compor um mercado de trabalho, que passa a ser avaliado a partir das dimensões de vida social livre, atendendo às determinações de mercado, enquanto ente subjetivo, em que tanto os ofertantes quanto os demandantes do trabalho o compreendem como uma mercadoria qualquer.

Nesse contexto, na concepção de Marx (1994), no processo de trabalho, a ação do homem diante da natureza opera uma transformação no objeto sobre o qual atua por meio de instrumentos de trabalho para a produção de mercadorias, e essa transformação está subordinada a um determinado fim. Assim, consideram-se três elementos componentes do processo de trabalho: a atividade adequada a um fim, isto é, o próprio trabalho, em geral assumindo um formato de mercadoria; o objeto de trabalho, ou seja, a matéria a que a ele se aplica, e os instrumentos ou meios do trabalho, os recursos sobre os quais se adquire o direito de dominar e até mercantilizar o trabalho do outro.

Com efeito, é relevante ressaltar que o processo de trabalho e seus componentes constituem categorias de análise, portanto abstrações teóricas por meio das quais é possível abordar e compreender certos aspectos da realidade objetiva, do mundo material. No caso desta pesquisa, os processos educativos de trabalho que formam, naturalmente, o cuidador de idoso numa perspectiva de educação em saúde ou das práticas das escolas. Essas instituições conseguem formar trabalhadores que fazem do cuidado, habilidade inerente e solidária em respeito ao outro, um serviço administrado e/ou vendido no mercado por entidades ou empresas que se tornam, ao longo do processo histórico, instrumento de modificação e posse sobre o trabalho, como um produto qualquer que se leva ao mercado.

Assim, diante do exposto, faz-se a seguinte indagação: o cuidador da pessoa idosa nasce partir de um processo educativo solidário com "valor de uso" ou de uma formação específica para o mercado? Responder a essa indagação não é tarefa fácil, entretanto, pretende-se discorrer nesta pesquisa como tem se desenvolvido o trabalho do cuidador de idosos e, a partir de então, fazer reflexões e/ou inferências. Tem-se, dessa forma, como propósito nesta pesquisa discutir o ofício de cuidador da pessoa idosa no desenvolvimento de suas habilidades frente às novas relações de trabalho, bem como as configurações mercadológicas em que o cuidado com o outro passa assumir um "valor de troca" ou define-se num trabalho qualquer oferecido no mercado.

Metodologicamente, trata-se de um estudo teórico com sustentação em referências das áreas, com articulações interdisciplinares a partir das temáticas envolvidas em que o próprio trabalho assume a centralidade das discussões. Espera-se, ao final, inferir uma compreensão com base nas reflexões e práticas dos profissionais de saúde que acompanham o 
cuidado em relação à pessoa idosa, sobre qual configuração o cuidado com o (a) idoso (a) tem mais se aproximado, se de um processo educativo e solidário de trabalho que apenas produz "valor de uso" ou de uma tendência mercadológica em que o cuidado como a pessoa idosa posta-se como um serviço, guardadas as suas devidas atenções.

\section{2 - O CUIDADO COM A PESSOA IDOSA COMO UM ELEMENTO FORMATIVO DE EDUCAÇÃO EM SAÚDE}

As estratégias de educação em saúde têm sido mecanismos bastante efetivos e promissores no cuidado com a pessoa idosa, seja pelo baixo custo agregado dos serviços, tendo em vista que se devem reunir responsáveis pelo cuidado, cuja base é a articulação de um trabalho educativo entre cuidador, paciente e família, seja pela atenção humanizada que se empreende às famílias e pacientes fragilizados com doenças de longa duração ou progressivas. Essas duas situações podem ser valoradas ou não, a depender de como se utilizam as habilidades em relação ao trabalho que se despende enquanto cuidador (BENJUMEA, 2004).

Nessa perspectiva, Freire (1987), afirma que a educação para a saúde é um processo que utiliza formas de aprendizagem que visam permitir aos indivíduos tomar decisões fundamentadas num processo ativo, crítico e transformador. Tem como intuito construir o saber de modo coletivo e não apenas transmitir informações, nem vislumbrar resultados onerosos para quem cuida. Visa também contribuir para melhorar a qualidade de vida dos indivíduos e da comunidade na qual estão inseridos sem ter, necessariamente, interesses onerosos envolvidos.

Dessa forma, o processo de educação consiste também na perspectiva de mudanças do sujeito do processo, o cuidador, na busca de melhorias de um cuidado qualificado com a pessoa idosa dependente que vive em domicílio. Nessa esteira, já se vislumbra a possiblidade de um processo formativo de trabalho.

Assim, segundo o Estatuto do Idoso, artigo 10 "Idoso é toda pessoa com idade igual ou superior a 60 (sessenta) anos”. (BRASIL, 2003, p.7). A criação desse estatuto possibilitou a regulamentação dos direitos da pessoa idosa, algo que era e ainda é bastante desrespeitado. Compreendemos, entretanto, que não basta criar as Leis, pois sua relevância não garante aplicação. É preciso haver uma mobilidade social do seguimento diretamente interessado em torno da sua efetividade.

Durante o processo de envelhecimento, a pessoa idosa vivencia situações diversas de fragilidades específicas da faixa etária, marcado por várias modificações anatômicas, fisiológicas, psicológicas que devem ser compreendidas por pessoas que atuam diretamente no cuidar, ou seja, em geral, formadas para isso. Nesse contexto, o conjunto das alterações necessitam de cuidado humanizado, sistematizado e individualizado para cada pessoa idosa dependente de cuidador.

O presente estudo vislumbra dar continuidade e aprofundar conhecimentos adquiridos nas nossas experiências sobre a temática do cuidado da saúde da pessoa idosa, na perspectiva de conhecer os possíveis impactos sobre preservação da autonomia de pessoas idosas dependentes, a partir das estratégias educativas junto aos cuidadores, buscando contribuir 
com a formação de pesquisadores e subsidiar ações e políticas de cuidado sistematizado e com atendimento qualificado a este grupo populacional (GOLDIN, 2002). Além disso, pretender-se discutir essa formação enquanto uma perspectiva de trabalho que ora se utiliza sem valor de uso, ora é levado ao mercado e produz valor de troca.

Entretanto, para além dessas discussões, destaca-se a sua relevância para a comunidade acadêmica e para a sociedade em geral, uma vez que permite verificar se os cuidadores de pessoas idosas dependentes desenvolvem os cuidados de maneira qualificada e se, conhecendo mais sobre seu envolvimento neste processo, bem como sobre as necessidades deste grupo, pode, a partir dos resultados, possibilitar mudanças necessárias, tais como uma formação mais profissional e socialmente valorizada.

Socialmente, é visível a necessidade de criação da rede de atenção para os cuidadores da pessoa idosa, uma vez que é fundamental assistir essa população que vem crescendo em seus diversos aspectos, durante a assistência domiciliar, a fim de proporcionar diretrizes para o cuidado necessário à pessoa idosa dependente de cuidados, enquanto um ambiente de conhecimentos e saberes articulados.

Segundo a Organização Mundial de Saúde (OMS) a educação em saúde é entendida como sendo uma combinação de ações e experiências de aprendizado planejada com o intuito de habilitar as pessoas a obterem controle sobre fatores determinantes e comportamentos de saúde.

Nessa perspectiva, o Ministério da Saúde define educação em saúde como:

Processo educativo de construção de conhecimentos em saúde que visa à apropriação temática pela população [...]. Conjunto de práticas do setor que contribui para aumentar a autonomia das pessoas no seu cuidado e no debate com os profissionais e os gestores, a fim de alcançar uma atenção de saúde de acordo com suas necessidades. (MINISTÉRIO DA SAÚDE, 2009. p. 22).

Reconhecendo que a educação é, antes de tudo, um processo de promoção humana, reafirmamos que é através das pessoas envolvidas no contexto educativo que se vislumbra mudança de hábitos e estilo de vida, a partir do aprendizado de práticas e orientações adequadas. De forma específica, nesse diálogo entre educação e saúde, está a relevância desta proposta no sentido de compreender que a educação em saúde, quando realizada permanentemente, se configura como mudança de hábitos e transformação no estilo de vida do indivíduo, de maneira que o mesmo passa a cuidar de si e, consequentemente, mudar o meio em que vive.

No entanto, para os limites da presente proposta, o processo de educação em saúde é de grande importância na prevenção de doenças e na melhoria da qualidade de vida das pessoas através das orientações necessárias capazes de influenciar mudanças de comportamentos, práticas e atitudes. No entendimento de Vasconcelos (2001), o termo educação em saúde vem sendo utilizado desde as primeiras décadas do século XX e, para sua melhor compreensão, um breve histórico sobre a saúde pública no Brasil contribui para esclarecer esse ponto. 
Segundo Andrade (2001), a educação em saúde tradicional, inicialmente chamada de Educação Sanitária, surge no Brasil a partir da necessidade de o Estado brasileiro controlar as epidemias de doenças infectocontagiosas que ameaçavam a economia agroexportadora do país durante a República Velha (1891-1930). Nesse período, a população brasileira era acometida por doenças que estavam relacionadas às precárias condições sanitárias e socioeconômicas em que se vivia.

Nesse sentido, a educação em saúde como estratégia educativa tem como um dos pontos de partida a perspectiva que reconhece a micropolítica do trabalho em saúde. (MERHY, 2007). Para tal pesquisador, diversas tecnologias podem estar envolvidas no trabalho em saúde, como as leves, que se referem às tecnologias de relações do tipo produção de vínculo, autonomização, acolhimento, gestão como uma forma de governar processos de trabalho. Assim sendo, a proposta do uso de tecnologias em saúde representa uma estratégia fundamental de processo educativo para o cuidador realizador das demandas dos cuidados da pessoa idosa dependente.

Para Girondi (2011), a educação em saúde é entendida como prática para a transformação dos modos de vida dos indivíduos e da coletividade e, consequentemente, para promover qualidade de vida e saúde. Portanto, faz-se necessário conhecer as estratégias de educação em saúde que estão sendo utilizadas para melhorar a assistência às pessoas idosas dependentes, a fim de identificar determinadas lacunas acerca do envelhecimento, como a carência de estudos sobre as atividades realizadas nos serviços de saúde que respondam às necessidades dos idosos e visem à promoção da saúde.

O envelhecimento da população é apontado como um dos problemas cruciais do século XXI. (CABRAL et al., 2013). As alterações demográficas e o aumento da esperança média de vida da população impõem diversos desafios aos indivíduos e à sociedade, traduzindo-se, a nível individual, no aumento da longevidade, e a nível coletivo, nas mudanças e deficiências das relações sociais e familiares, bem como pelas implicações econômicas. (POCINHO, 2014).

O crescimento da população idosa é um fenômeno de abrangência mundial. Estudos demográficos sobre essa temática evidenciam um expressivo crescimento da população acima dos 60 anos, e as projeções para o ano de 2050 sugerem que haverá um total de, aproximadamente, dois bilhões de pessoas com 60 anos ou mais no mundo, sendo que 80\% dessas pessoas se encontrarão nos países em desenvolvimento. (BRASIL, 2006).

Assim, a possibilidade da elevada prevalência de doenças crônico-degenerativas somada à decorrência de pluripatogenia (evidência de mais de uma doença concomitante) e à carência de respostas mais coerentes dos sistemas de saúde, uma vez que estes, de acordo com Mendes (2010), são as respostas sociais deliberadas às necessidades de saúde da população.

A Política Nacional de Saúde do Idoso (PNSI) - Portaria do Ministério da Saúde $\mathrm{n}^{\circ}$ 1.395, de 1999, instrumento de que o setor saúde passa a dispor, tem como propósito basilar a promoção do envelhecimento saudável, a preservação e/ou a melhoria, ao máximo possível, da capacidade funcional dos idosos, a prevenção de doenças, a recuperação da saúde daqueles que adoecem e a reabilitação daqueles que venham a ter a sua capacidade funcional 
restringida, de modo a garantir-lhes permanência no meio em que vivem, exercendo de forma independente suas funções na sociedade. (GORDILHO et al, 2000).

Segundo o Ministério da Saúde (2007), uma grande parcela de profissionais da área da saúde e cuidadores sem esclarecimentos enfrentam a dúvida do que fazer, bem como do tipo de apoio que necessitam para enfrentar as patologias decorrentes do processo de envelhecimento. Em se tratando de doença neurológica crônico-degenerativa, traz consigo dúvidas em relação ao manejo do doente, afetando aspectos de ordem pessoal, emocional, financeira e social do paciente e seus familiares.

O que se percebe é que as demandas dos cuidados da pessoa idosa são diversas, tendo em vista algumas particularidades desse público, esses cuidados incluem uma assistência qualificada por parte do cuidador que convive em seus lares e envolve até os familiares. Netto (1996) afirma que cuidar é o ato de assistir alguém ou prestar-lhe serviços quando necessita. É uma atividade complexa, com dimensões éticas, psicológicas, sociais e demográficas e que também tem seus aspectos clínicos, técnicos e comunitários.

Sabemos que existem Leis no Brasil que têm por finalidade proteger a pessoa idosa, bem como garantir sua participação na comunidade, além de defender sua dignidade, zelar pelo seu bem-estar e garantir o direito à vida. Entretanto, é percebido que o sistema terapêutico para essa população é deficitário, uma vez que a população citada depende em algum momento de cuidados de outras pessoas.

Nesse sentido, é necessário reunir ações de educação, promoção da saúde, prevenção de doenças evitáveis, cuidado precoce e reabilitação de agravos através de uma Rede de Cuidado, capacitação de pessoas que prestam cuidados à pessoa idosa com a finalidade de garantir qualidade na assistência, tendo em vista que se trata da articulação de cuidados que fazem parte de um processo em que o foco principal não é, necessariamente, a doença e sim a pessoa, através de proteção para melhoria do sofrimento vivido.

Sendo assim, justifica-se a necessidade de criação de Curso de Capacitação para os cuidadores da pessoa idosa dependente, uma vez que é fundamental assistir a essa população em seus diversos aspectos durante a assistência domiciliar, a fim de proporcionar diretrizes para o cuidado necessário à pessoa idosa dependente de cuidados, tendo em vista que sempre fazem parte da rotina do cuidador a assistência geral da saúde, o conforto, a higiene, a educação e a promoção da saúde.

Dessa forma, surge a necessidade de articular capacitações, orientações, oficinas para os cuidadores sobre a necessidade de cuidados direcionados à pessoa idosa dependente. Espera-se que essas ações possibilitem reflexão para melhoria dessa forma de cuidado, além de permitir conhecer os desafios enfrentados durante a realização do cuidado à pessoa idosa e elaborar um estudo junto ao processo de gestão do trabalho com o cuidador, visando o controle das ações de cuidado qualificado.

Assim, acreditamos que este estudo possa promover ou possibilitar mudanças positivas nas estratégias de saúde/educação para os cuidados com a pessoa idosa dependente por meio da transformação das práticas de cuidados relacionadas aos aspectos gerais da saúde, conforto, higiene, educação e promoção à saúde através do cuidado qualificado. Além disso, 
torna-se necessário mobilizar a discussão em torno do contexto das classes hospitalares e domiciliares; promover a discussão da educação para a saúde em todas as áreas possíveis da sociedade, bem como sensibilizar as instituições públicas e privadas competentes e os meios de comunicação, sobre a necessidade de projetos que incentivem a educação para uma sociedade mais saudável.

Diante desse contexto, a assistência à pessoa idosa dependente deve ser vista para além da solidariedade das pessoas e das famílias, embora essas continuem sendo as bases necessárias da sensibilidade humana em relação ao outro, consubstanciando-se em uma relação de trabalho em que seja levada em conta a formação, as habilidades, os custos formativos, as diretrizes legais regulatórias e as políticas públicas. Além disso, é imprescindível a observância do Estado enquanto uma dimensão eivada de perspectiva para políticas públicas, em que pese o cuidado, enquanto serviço de saúde que possa ser executado, seja nos domicílios, seja nos empreendimentos de saúde públicos ou particulares a bem da população idosa.

\section{3 - METODOLOGIA}

A metodologia consiste no caminho que define uma lógica de raciocínio que possibilita compreender a forma científica a partir da qual a pesquisa é elaborada sob a égide de determinados critérios (GIL,1999). Ainda segundo o mesmo autor, os procedimentos metodológicos se valem de instrumentos diversos como levantamentos bibliográficos de referência da área para sustentação teórica da pesquisa, levantamento de dados e informações nos ambientes que arquivam e guardam prontuários dos procedimentos, bem como entrevistas semiestruturadas, além de outros procedimentos que se fizerem necessários no decorrer do processo de pesquisa. Assim, este trabalho consiste, em parte, das discussões que gerarem nossa proposta de pesquisa para o acesso ao Programa de Pós-Graduação GESTEC, embora esse tenha sido apenas um dos objetivos apontados na pesquisa inicial.

Trata-se, portanto, de um estudo de campo, exploratório, de caráter qualitativo. Nesse tipo de estudo, o pesquisador procura entender os fenômenos e compreender o significado das coisas segundo a perspectiva dos participantes e da situação estudada e, a partir daí, situa sua interpretação dos fenômenos estudados. (TURATO, 2005).

O campo do estudo define-se no município de Feira de Santana-BA, localizado a 110 $\mathrm{km}$ da capital Salvador. Considerada a segunda maior cidade do Estado da Bahia/Brasil com uma área territorial $1.363 \mathrm{~km}^{2}$ e uma população estimada de 591.707 habitantes, sendo 8,7\% a população de idosos, conforme censo IBGE (2011).

A Secretaria Municipal de Saúde, através da Atenção Básica, dispõe de 132 equipes atuando nas Unidades de Saúde da Família (USF). Esta pesquisa vem sendo desenvolvida no bairro Santo Antônio dos Prazeres no município de Feira de Santana, Bahia, onde constam duas equipes de Saúde da Família, que atuam desde 2003. As equipes têm 2.008 (dois mil e oito) famílias cadastradas, com uma média de 380 idosos (as) acompanhados (as) e, aproximadamente, 12 idosos (as) acamados (as) e domiciliados (as). 


\section{4 - ANÁLISES E RESULTADOS}

A vida humana consubstancia-se em um processo contínuo em que as experiências vividas compõem a sua história e alimenta um processo existencial que possibilita facilitar a vivência das gerações que se sucedem. Dessa forma, o ser humano tal como os demais seres vivos tem um ciclo vital relevante na construção da memória social: nascem, crescem, desenvolvem-se, tornam-se adultos, envelhecem e morrem. O envelhecimento, especialmente, tem marcado relevantes desafios não somente para a memória social, mas, principalmente, para registros, estudos e pesquisas sobre a evolução e vida da pessoa idosa. O envelhecimento populacional é um fenômeno global.

Essa mudança demográfica é consequência do acentuado declínio na taxa de fertilidade e do aumento da expectativa de vida observados, principalmente, a partir de meados do século XX (LIMA, 2011), fato que vem se projetando a passos largos.

De acordo com os dados do Instituto Brasileiro de Geografia e Estatística (IBGE, Censo 2010), ao longo dos últimos 50 anos, a população brasileira quase triplicou: passou de 70 milhões, em 1960, para 190,7 milhões, em 2010. O crescimento do número de idosos, no entanto, foi ainda maior. Em 1960, 3,3 milhões de brasileiros tinham 60 anos ou mais e representavam 4,7\% da população. Em 2000, 14,5 milhões, ou seja, 8,5\% dos brasileiros estavam nessa faixa etária. Na última década, o salto foi grande, e em 2010, a representação passou para 10,8\% da população (20,5 milhões).

Com a mudança no perfil demográfico da população, há um crescimento considerável na prevalência de doenças crônicas não transmissíveis (DNTs). O Ministério da Saúde afirma que as doenças crônicas tendem a se manifestar de forma mais expressiva nas pessoas idosas, podendo comprometer de forma significativa a qualidade de vida dessas, além de ser responsável pelas principais causas de morbimortalidade nessa população (BRASIL, 2006).

No processo do cuidar, identificamos os princípios, os valores e as atitudes que fazem da vida um bem-viver e das ações que demandam um reto agir (BOFF, 2000). Neste sentido, a ação de saúde focada na lógica da produção do cuidado traduz-se no processo baseado nos problemas, nas necessidades e na qualidade de vida dos usuários, pois além de produzir os procedimentos associados direcionam também a atenção nas relações humanas, na produção de vínculo, no acolhimento, e na cor-responsabilização do usuário no cuidado. (MATSUMOTO, 2012). Daí a necessidade da sistematização organizada do cuidado individualizado, em especial, na emergente assistência à pessoa idosa.

Compreendendo que os cuidados aos seres humanos são necessários, a fim de permitir a continuidade da vida das pessoas, dos grupos e das sociedades, pressupõe-se que sua origem esteja relacionada a qualquer pessoa que ajude outra a ter garantida a manutenção da sua vida e não a um ofício ou profissão (COLLIÈRE, 1999).

Essa pesquisa tem um relevo especial pelo crescimento da população acima dos 60 anos, sendo a faixa etária que mais cresce nas últimas décadas, e por saber que as demandas dos cuidados da pessoa idosa são diversas, tendo em vista algumas particularidades desse público. Esse processo de cuidar salta do acolhimento pessoal e familiar para uma 
necessidade formativa como um processo de trabalho, cuja dinâmica e habilidades precisam ser desenvolvidas a partir de uma medida social necessária em trabalho despendido em favor do estado ou de outros usuários.

Nesse contexto, durante nossa experiência de três anos na referência do idoso, após registrar uma média de 30 visitas a pessoas idosas acamadas, pudemos observar que dentre todos os casos de visitas, apenas um se apresentava dentro do protocolo do cuidado, enquanto procedimento formativo de trabalho, seja para o cuidador seja para a pessoa idosa.

Com efeito, esse tema aponta para a necessidade de criação da rede de atenção para os cuidadores da pessoa idosa, uma vez que é fundamental assistir essa população em seus diversos aspectos durante a assistência domiciliar, em especial, aqueles de renda menor que se constituem na sua maioria, a fim de proporcionar diretrizes para o cuidado necessário à pessoa idosa dependente de cuidados, tendo em vista, que sempre fazem parte da rotina do cuidador os cuidados gerais da saúde, conforto, higiene, alimentação, educação e promoção da saúde.

A ocupação de cuidador de pessoas idosas e dependentes integra a Classificação Brasileira de Ocupações (CBO, 2017), um documento que retrata a realidade das profissões do mercado de trabalho brasileiro, como um profissional que "cuida a partir dos objetivos estabelecidos por instituições especializadas ou responsáveis diretos, zelando pelo bem-estar, saúde, alimentação, higiene pessoal, educação, cultura, recreação e lazer da pessoa assistida". (CBO, 2017).

A profissão de Cuidador de Idosos, Crianças, Pessoa com Deficiência e Doenças Raras foi regulamentada e a proposta Projeto Lei da Câmara (PLC 11/2016) aprovada no dia 07 de fevereiro de 2018, pela Comissão de Assuntos Sociais (CAS), visando criar empregos e garantir os direitos trabalhistas da categoria. Essa regulamentação estabelece que a atividade deve ser exercida por pessoas com o ensino fundamental, com curso de qualificação na área, além de idade mínima de 18 anos, bons antecedentes criminais e atestados de aptidão física e mental. Entre as atribuições, o cuidador deve buscar a autonomia e a independência e zelar pelo bem-estar, saúde, alimentação, higiene pessoal e recreação da pessoa que acompanha e dá assistência.

De acordo com o Projeto de Lei do Senado (PLS) 284/11, cuidador de idoso é o profissional que, no âmbito domiciliar ou de instituição de longa permanência para idosos, desempenha funções de acompanhamento, como a prestação de apoio emocional e na convivência social do idoso; o auxílio e o acompanhamento na realização de rotinas de higiene pessoal e ambiental e de nutrição; os cuidados de saúde preventivos, a administração de medicamentos de rotina e outros procedimentos de saúde; e o auxílio e o acompanhamento no deslocamento do idoso.

Reconhecendo que o Parecer Técnico de nº 582, de 17 de julho de 2018, do Conselho Federal de Enfermagem (COFEN), normatiza a atuação do (a) enfermeiro (a) nos cursos de formação de Cuidadores de Idosos, esse parecer determina que cabe ao (a) enfermeiro (a) ministrar conteúdos relacionados a aspectos gerais da saúde, conforto, higiene, educação e promoção da saúde. A normativa proíbe que os (as) enfermeiros (as) ensinem práticas de 
enfermagem que exijam a aplicação de conhecimentos técnicos-científicos, tanto em aulas teóricas como em atividade de estágios em cursos que visem a formação de cuidadores.

Percebe-se que a função do cuidador da pessoa idosa é de acompanhar e auxiliar a pessoa a se cuidar, por meio de orientações gerais da saúde, conforto, higiene, educação e promoção da saúde, fazendo pela pessoa somente as atividades que ela não consiga fazer sozinha, além de exercer seu trabalho com conhecimentos adequados e específicos na perspectiva de garantir cuidados especiais com segurança.

É preciso, sobretudo, entender que o trabalho realizado pelo cuidador é caracterizado como trabalho necessário para a sobrevivência desse indivíduo que carece de cuidados diários e para isso é importante ressaltar as demandas desses cuidadores que têm sua atividade como principal fonte de renda e/ou motivação financeira.

É fundamental compreender também que cuidar se trata de uma tarefa nobre, porém complexa, percebida em alguns momentos com déficit no conhecimento da terapêutica e manejo especializados.

\section{5 - CONSIDERAÇÕES FINAIS}

A partir das discussões, reflexões e inferências proporcionadas por esta pesquisa, passamos a vislumbrar o cuidador da pessoa idosa não só como uma "pessoa" de compromisso individual ou coletivo resultante de um processo educativo solidário que traz em seu bojo não apenas "valor de uso" enquanto um trabalho ou ajuda desinteressada de remunerações ou promoções que não produz efeitos mercadológicos, porque as pessoas executam sem troca equivalente a um benefício qualquer.

Por outro lado, entretanto, observamos que a formação específica se torna cada vez mais necessária, o que acaba produzindo algo de valor mediado pela sociedade ou pelo mercado, com remunerações variadas designadas pelo nível de formação que se aloca em cada pessoa ou profissional cuidador.

A problematização sobre essa sensível profissão tão necessária não é uma questão das mais simples, entretanto, infere-se que o trabalho do cuidador de idosos ou o ofício de cuidador de idoso tem se desenvolvido com base nas habilidades de um contexto de amplas dimensões possibilitadas nas relações de trabalho, cuja dinâmica se observa nas configurações mercadológicas em que até mesmo a solidariedade das relações não consegue dar conta do cuidado com o outro. Paradoxalmente, o trabalho com o idoso formatado enquanto mercadoria, passa a assumir um valor de troca ou um trabalho como outro qualquer oferecido no mercado.

É mister que não se tem a pretensão de resolver discussões de tal relevância apenas com esta pesquisa, entretanto, espera-se contribuir para uma compreensão com base nas reflexões e práticas dos profissionais de saúde que acompanham o cuidado em relação ao idoso. Além de entender que o cuidado com a pessoa idosa tem cada vez mais se aproximado de uma tendência mercadológica que se configura como um serviço, guardadas as suas devidas atenções, uma mercadoria (serviço) como outra qualquer, um valor de troca. 


\section{6 - REFERÊNCIAS}

ANDRADE, O. M. de. SUS passo a passo: normas, gestão e financiamento. São Paulo -Sobral: Editora Hucitec: Edições Uva, 2001.

BOFF, L. Saber Cuidar: ética do humano - compaixão pela terra. Petrópolis: Vozes, 2000.

BRASIL. Ministério da Saúde. Conselho Nacional de Saúde. Resolução n. 466, de 12 de dezembro de 2012. Aprova diretrizes e normas regulamentadoras de pesquisas envolvendo seres humanos. Brasília, Diário Oficial da União, 12 dez. 2012. Disponível em: http://conselho. saude.gov.br/ultimas_noticias/2013/06_jun_14_publicada_resolucao.html.

BRASIL. Estatuto do Idoso. Lei $n^{\circ} 10.741$, de 1 de Outubro de 2003. Dispõe sobre o Estatuto do Idoso e dá outras providências. Diário Oficial da União. 3 Out 2003. Disponível em: https:// bvsms.saude.gov.br/bvs/publicacoes/estatuto_idoso_3edicao.pdf.

BRASIL. Política Nacional de Saúde do Idoso. Portaria do Ministério da Saúde no 1.395 de 1999.

BRASIL. Ministério da Saúde (MS). Secretaria-Executiva. Secretaria de Gestão do Trabalho e da Educação na Saúde. Glossário temático: gestão do trabalho e da educação na saúde. Brasília: Editora do Ministério da Saúde; 2009. (Série A. Normas e Manuais Técnicos).

BRASIL. Ministério da Saúde (BR), Secretaria de Atenção a Saúde, Departamento de Atenção Básica. Envelhecimento e saúde da pessoa idosa. Brasília (DF); 2007. (Cadernos de Atenção Básica; 19). Disponível em: http://bvsms.saude. gov.br/bvs/publicacoes/abcad19.pdf.

BRASIL. Ministério da Saúde (MS). Envelhecimento e saúde da pessoa idosa. Brasília: MS; 2006.

BRASÍLIA. Secretaria Especial de Editoração e Publicações do Senado Federal - PROJETO DE LEI DO SENADO N 284, DE 2011 - Dispõe sobre o exercício da profissão de cuidador de idoso. Brasília-DF OS: 12400/2011.

BENJUMEA C. C. Cuidado familiar en condiciones crónicas: una aproximación a la literatura. Revista Texto e Contexto Enfermagem, 2004 janeiro-março; 13(1): 137-46.

CABRAL. M. V. et al. Processos de Envelhecimento em Portugal: Uso do tempo, redes sociais e condições de vida. Lisboa: Fundação Francisco Manuel dos Santos, 2013.

\section{CLASSIFICAÇÃO BRASILEIRA DE OCUPAÇÕES. Ministério do Trabalho, 2017.}

CONSELHO FEDERAL DE ENFERMAGEM. Resolução Cofen $n^{0}$ 582/2018. Veda a participação do Enfermeiro no ensino de práticas de Enfermagem que exija aplicação de conhecimentos técnico científicos em atividades de formação de Cuidador de Idosos. Disponível em: < http://www.cofen.gov.br/resolucao-cofen-no-582-2018_64391.html>. Acesso em 28 jan. 2019. 
COLLIÉRE MF. Promover a vida: da prática da mulher de virtude aos cuidados de enfermagem. 4a ed. Coimbra (Po): Ledil; 1999.

FREIRE, P. Pedagogia do oprimido. Rio de Janeiro: Paz e Terra, 1987.

GIRONDI, J.B.R.; SANTOS, S.M.A. Deficiência física em idosos e acessibilidade na atenção básica em saúde: revisão integrativa da literatura. Revista Gaúcha de Enfermagem, 2011; 32(2):378-384.

GIL, A. C. Métodos e técnicas de pesquisa social. 5.ed. São Paulo: Atlas, 1999.

GOLDIN, JR. Bioética e envelhecimento. In: Freitas EV, Py L, Neri AL, Cançado FAX, Doll J, Gorzoni ML, organizadores. Tratado de geriatria e gerontologia. Rio de Janeiro: Guanabara Koogan; 2002.

GORDILHO A, et al. Desafios a serem enfrentados no terceiro milênio pelo setor saúde na atenção integral ao idoso. Rio de Janeiro (RJ): UnATI/UERJ; 2000.

IBGE - INSTITUTO BRASILEIRO DE GEOGRAFIA E ESTATÍSTICA. Censo demográfico 2010: características da população e dos domicílios. Resultados do Universo. Rio de Janeiro, 2011 .

LIMA. Costa, M.F. Estudo de Coorte de Idosos de Bambuí (1997-2008). Cad. Saúde Pública, 27(Sup 3): S324-S325. Rio de Janeiro (RJ), 2011.Recuperado em 20 junho, 2019, de: http:// www.scielosp.org/pdf/csp/v27s3/pt 01.pdf.

MATSUMOTO, D. Y. Cuidados Paliativos: conceito, fundamentos e princípios. In: CARVALHO, R. T.; PARSONS, H. A. (Org.) Manual de Cuidados Paliativos. São Paulo: Academia Nacional de Cuidados Paliativos (ANCP), 2012. p.23-30.

MARX, K. O Capital. 14. Ed. São Paulo: Difel, 1994. V.1.

MENDES, E.V. As redes de atenção à saúde. Ciência \& Saúde Coletiva, 15, 2010. Recuperado em 20 junho, 2019.

MERHY, Emerson Elias. Saúde: a cartografia do trabalho vivo. São Paulo: Hucitec, 2007.

NETTO, M.P. Gerontologia. Editora Atheneu. São Paulo, 1996.

POCINHO, Ricardo, S. P. Mayores en contextos de aprendizaje: Caracterización y efectos psicológicos en los alumnos de las Universidades de Mayores en Portugal. Tese de doutoramento em Psicogerontologia. Universitat de València, 2014.

TURATO, E. R. Métodos qualitativos e quantitativos na área da saúde: definições, diferenças e seus objetos de estudo. Rev. Saúde Pública. V. 39, n3. 2005, p 507-514.

VASCONCELOS, E.M. Educação popular nos serviços de saúde. 3a Ed. São Paulo: Editora Hucitec, 2001. 